\title{
A generalização dos quatérnios de Narayana
}

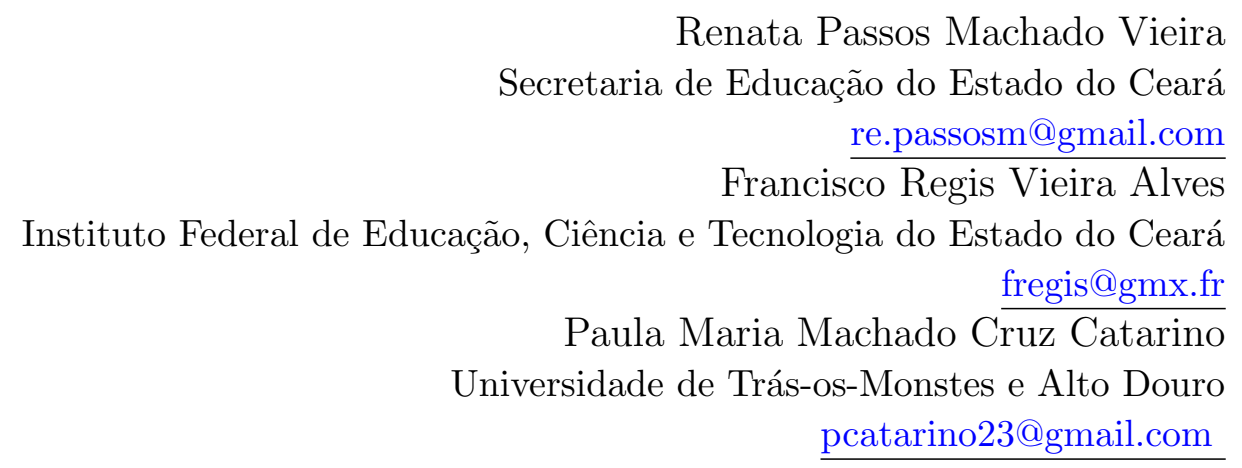

\section{Resumo}

A pesquisa retrata uma discussão matemática em torno dos quatérnios de Narayana e a sua generalização para os números inteiros não positivos. Como resultados apresentados, tem-se que a partir do estudo da definição desses números, são investigadas algumas propriedades matemáticas. Com isso, retrata-se a sua forma matricial, função geradora, fórmula de Binet e dentre outros aspectos matemáticos inerentes aos quatérnios de Narayana.

Palavras-chave: forma matricial, fórmula de Binet, quatérnios, sequência de Narayana.

\section{Abstract}

The research portrays a mathematical discussion around Narayana quaternions and their generalization to non-positive integers. As presented results, it has been that from the study of the definition of these numbers, some mathematical properties are investigated. With that, it portrays its matrix form, generating function, Binet formula and among other mathematical aspects inherent to Narayana's quaternions.

Keewords: matrix form, Binet's formula, quaternions, Narayana sequence.

\section{Introdução}

Introduzida pelo matemático indiano Narayana Pandita (1340-1400), a sequência de Narayana é conhecida pela problemática das vacas e bezerros [1,5]. Com isso, tem-se a notação $N_{n}$, representando essa sequência. Tão logo, a sequência de Narayana é do 
tipo numérica, de terceira ordem, linear e recorrente. Assim, apresenta-se a seguinte relação de recorrência $N_{n}=N_{n-1}+N_{n-3}, n \geqslant 3, N_{0}=0, N_{1}=N_{2}=1$.

O seu polinômio característico é dado pela equação $x^{3}-x^{2}-1=0$, possuindo como soluções duas raízes complexas e uma raiz real. O valor real da raiz com valor aproximado de 1,46, é representado pela proporção do super-ouro. Ao tudo, outros trabalhos retratam alguns aspectos matemáticos e históricos inerentes à essa sequência $[1,2]$.

Com o viés de realizar uma evolução matemática dessa sequência, estuda-se o seu processo de complexificação, com os números quatérnios. Desenvolvidos por Willian Rowan Hamilton (1805-1865) por volta de 1843, os quatérnios são números hipercomplexos, estudados na álgebra abstrata. Tão logo, existe duas estruturas quaterniônicas, sendo essas: os quatérnios sobre $\mathbb{R}$ (que possuem componentes reais), e os biquatérnios sobre o $\mathbb{C}$ (que possuem componentes complexas) [9, 10].

Dessa forma, esses números são descritos por:

$$
q=a+b i+c j+d k
$$

onde $a, b$ e $c$ são números reais, e $i, j$ e $k$ a parte ortogonal na base $\mathbb{R}^{3}$. Ressalta-se que, com base em Horadam (1993) [8], existe ainda operações que podem ser realizadas para esses números, bem como os respetivos produtos quaterniônicos: $i^{2}=j^{2}=k^{2}=$ $-1, i j=k=-i j, j k=i=-k j$ e $k i=j=-i k$.

Em alguns trabalhos, associam-se os quatérnios a outras sequências lineares e recorrentes, como é o caso de Padovan e Perrin [4], Pell-Padovan [13] e Fibonacci [3, 8, 6, 7, 11]. Com base nisso, esta pesquisa apresenta os quatérnios de Narayana, juntamente com a sua generalização para os números inteiros e outras propriedades matemáticas discutidas a seguir.

\section{Os quatérnios de Narayana}

Nesta seção, são estudados os quatérnios de Narayana, abordando os seus respectivos aspectos matemáticos.

Definição 2.1. Para $n \geqslant 0$, os quatérnios de Narayana são definidos por:

$$
Q N_{n}=N_{n}+i N_{n+1}+j N_{n+2}+k N_{n+3} .
$$

Definição 2.2. A fórmula de recorrência dos quatérnios de Narayana, é dada por:

$$
Q N_{n}=Q N_{n-1}+Q N_{n-3}
$$

onde $n \geqslant 3, n \in \mathbb{N}$. 
Teorema 2.3. A função geradora dos quatérnios de Narayana, $Q N_{n}$, é dada por:

$$
g\left(Q N_{n}, x\right)=\frac{i+j+k+(1+k) x+(j+k) x^{2}}{\left(1-x-x^{3}\right)} .
$$

Demonstração. Com base na função:

$$
g\left(Q N_{n}, x\right)=Q N_{0}+Q N_{1} x+Q N_{2} x^{2}+\ldots+Q N_{n} x^{n}+\ldots
$$

Pode-se realizar a multiplicação dessa função por $x$ e $x^{3}$, resultando:

$$
\begin{aligned}
x g\left(Q N_{n}, x\right) & =Q N_{0} x+Q N_{1} x^{2}+Q N_{2} x^{3}+\ldots+Q N_{n-1} x^{n}+\ldots \\
x^{3} g\left(Q N_{n}, x\right) & =Q N_{0} x^{3}+Q N_{1} x^{4}+Q N_{2} x^{5}+\ldots+Q N_{n-3} x^{n}+\ldots
\end{aligned}
$$

Realizando $g\left(Q N_{n}, x\right)-x g\left(Q N_{n}, x\right)-x^{3} g\left(Q N_{n}, x\right)$, tem-se que:

$$
\begin{aligned}
\left(1-x-x^{3}\right) g\left(Q N_{n}, x\right) & =Q N_{0}+\left(Q N_{1}-Q N_{0}\right) x+\left(Q N_{2}-Q N_{1}\right) x^{2} \\
\left(1-x-x^{3}\right) g\left(Q N_{n}, x\right) & =i+j+k+(1+k) x+(j+k) x^{2} \\
g\left(Q N_{n}, x\right) & =\frac{i+j+k+(1+k) x+(j+k) x^{2}}{\left(1-x-x^{3}\right)} .
\end{aligned}
$$

Teorema 2.4. A fórmula de Binet dos quatérnios de Narayana, com $n \in \mathbb{Z}$, é dada por:

$$
Q N_{n}=A \alpha_{q} x_{1}^{n}+B \beta_{q} x_{2}^{n}+C \gamma_{q} x_{3}^{n},
$$

em que $x_{1}, x_{2}, x_{3}$ são as raízes do polinômio característico $x^{3}-x^{2}-1=0$,

$$
\begin{aligned}
A & =\frac{\left(x_{2}-1\right)\left(x_{3}-1\right)}{\left(x_{1}-x_{2}\right)\left(x_{1}-x_{3}\right)}, B=\frac{\left(x_{1}-1\right)\left(x_{3}-1\right)}{\left(x_{2}-x_{1}\right)\left(x_{2}-x_{3}\right)}, C=\frac{\left(x_{1}-1\right)\left(x_{2}-1\right)}{\left(x_{3}-x_{1}\right)\left(x_{3}-x_{2}\right)}, \\
\alpha_{q} & =\left(1+x_{1} i+x_{1}^{2} j+x_{1}^{3} k\right), \beta_{q}=\left(1+x_{2} i+x_{2}^{2} j+x_{2}^{3} k\right), \gamma_{q}=\left(1+x_{3} i+x_{3}^{2} j+x_{3}^{3} k\right) .
\end{aligned}
$$

Demonstração. Por meio da fórmula de Binet $F N_{n}=\alpha x_{1}^{n}+\beta x_{2}^{n}+\gamma x_{3}^{n}$ e da recorrência dos quatérnios de Narayana $Q N_{n}=N_{n}+i N_{n+1}+j N_{n+2}+k N_{n+3}$, com os valores iniciais $Q N_{0}=i+j+k, Q N_{1}=1+i+j+2 k$ e $Q N_{2}=1+i+2 j+3 k$, é possível obter o seguinte sistema de equações:

$$
\begin{cases}\alpha+\beta+\gamma & =i+j+k \\ \alpha x_{1}+\beta x_{2}+\gamma x_{3} & =1+i+j+2 k \\ \alpha x_{1}^{2}+\beta x_{2}^{2}+\gamma x_{3}^{2} & =1+i+2 j+3 k\end{cases}
$$

ReviSeM, Ano 2021, No. 3, 12-22 
Resolvendo o sistema, tem-se que:

$$
\begin{aligned}
& \alpha=\frac{(1+i+2 j+3 k)+\left(-x_{2}-x_{3}\right)(1+i+j+2 k)+x_{2} x_{3}(i+j+k)}{x_{1}^{2}-x_{1} x_{2}-x_{1} x_{3}+x_{2} x_{3}}, \\
& \beta=\frac{(1+i+2 j+3 k)+\left(-x_{1}-x_{3}\right)(1+i+j+2 k)+x_{1} x_{3}(i+j+k)}{x_{2}^{2}-x_{2} x_{3}-x_{1} x_{2}+x_{1} x_{3}}, \\
& \gamma=\frac{(1+i+2 j+3 k)+\left(-x_{1}-x_{2}\right)(1+i+j+2 k)+x_{1} x_{2}(i+j+k)}{x_{3}^{2}+x_{1} x_{2}-x_{1} x_{3}-x_{2} x_{3}} .
\end{aligned}
$$

Através das relações de Girard: $x_{1} x_{2} x_{3}=1, x_{1}+x_{2}+x_{3}=1$ e $x_{1} x_{2}+x_{2} x_{3}+x_{1} x_{3}=0$, é fácil ver que:

$$
\begin{aligned}
\alpha & =\frac{\left(x_{2} x_{2}-x_{2}-x_{3}+1\right)}{\left(x_{1}-x_{2}\right)\left(x_{1}-x_{3}\right)}\left(1+x_{1} i+x_{1}^{2} j+x_{1}^{3} k\right)=\frac{\left(x_{2}-1\right)\left(x_{3}-1\right)}{\left(x_{1}-x_{2}\right)\left(x_{1}-x_{3}\right)}\left(1+x_{1} i+x_{1}^{2} j+x_{1}^{3} k\right) \\
& =A\left(1+x_{1} i+x_{1}^{2} j+x_{1}^{3} k\right), \\
\beta & =\frac{\left(x_{1} x_{3}-x_{1}-x_{3}+1\right)}{\left(x_{2}-x_{1}\right)\left(x_{2}-x_{3}\right)}\left(1+x_{2} i+x_{2}^{2} j+x_{2}^{3} k\right)=\frac{\left(x_{1}-1\right)\left(x_{3}-1\right)}{\left(x_{2}-x_{1}\right)\left(x_{2}-x_{3}\right)}\left(1+x_{2} i+x_{2}^{2} j+x_{2}^{3} k\right) \\
& =B\left(1+x_{2} i+x_{2}^{2} j+x_{2}^{3} k\right), \\
\gamma & =\frac{\left(x_{1} x_{2}-x_{1}-x_{2}+1\right)}{\left(x_{3}-x_{1}\right)\left(x_{3}-x_{2}\right)}\left(1+x_{3} i+x_{3}^{2} j+x_{3}^{3} k\right)=\frac{\left(x_{1}-1\right)\left(x_{2}-1\right)}{\left(x_{3}-x_{1}\right)\left(x_{3}-x_{2}\right)}\left(1+x_{3} i+x_{3}^{2} j+x_{3}^{3} k\right) \\
& =C\left(1+x_{3} i+x_{3}^{2} j+x_{3}^{3} k\right) .
\end{aligned}
$$

Definindo $\alpha_{q}=\left(1+x_{1} i+x_{1}^{2} j+x_{1}^{3} k\right), \beta_{q}=\left(1+x_{2} i+x_{2}^{2} j+x_{2}^{3} k\right), \gamma_{q}=\left(1+x_{3} i+\right.$ $\left.x_{3}^{2} j+x_{3}^{3} k\right), A=\frac{\left(x_{2}-1\right)\left(x_{3}-1\right)}{\left(x_{1}-x_{2}\right)\left(x_{1}-x_{3}\right)}, B=\frac{\left(x_{1}-1\right)\left(x_{3}-1\right)}{\left(x_{2}-x_{1}\right)\left(x_{2}-x_{3}\right)}$ e $C=\frac{\left(x_{1}-1\right)\left(x_{2}-1\right)}{\left(x_{3}-x_{1}\right)\left(x_{3}-x_{2}\right)}$, é fácil ver que:

$$
\alpha=A \alpha_{q}, \beta=B \beta_{q}, \gamma=C \gamma_{q}
$$

A forma matricial dos quatérnios de Narayana é realizada de acordo com o trabalho [12], em que retrata a matriz geradora da sequência de Narayana, segundo uma generalização dos seus respectivos coeficientes da sua fórmula de recorrência. 
Propriedade 2.5. Para $n \geqslant 3$ e $n \in \mathbb{N}$, a forma matricial dos quatérnios de Narayana é dada por:

$$
\begin{aligned}
{\left[\begin{array}{lll}
1 & 1 & 0 \\
0 & 0 & 1 \\
1 & 0 & 0
\end{array}\right]^{n}\left[\begin{array}{ccc}
Q N_{1} & Q N_{0} & Q N_{-1} \\
Q N_{-1} & Q N_{-2} & Q N_{-3} \\
Q N_{0} & Q N_{-1} & Q N_{-2}
\end{array}\right] } & =\left[\begin{array}{ccc}
N_{n+1} & N_{n} & N_{n-1} \\
N_{n-1} & N_{n-2} & N_{n-3} \\
N_{n} & N_{n-1} & N_{n-2}
\end{array}\right]\left[\begin{array}{ccc}
Q N_{1} & Q N_{0} & Q N_{-1} \\
Q N_{-1} & Q N_{-2} & Q N_{-3} \\
Q N_{0} & Q N_{-1} & Q N_{-2}
\end{array}\right] \\
& =\left[\begin{array}{lll}
Q N_{n+1} & Q N_{n} & Q N_{n-1} \\
Q N_{n-1} & Q N_{n-2} & Q N_{n-3} \\
Q N_{n} & Q N_{n-1} & Q N_{n-2}
\end{array}\right] .
\end{aligned}
$$

Demonstração. Pelo princípio da indução finita, tem-se que para $n=3$ :

$$
\begin{array}{r}
{\left[\begin{array}{lll}
1 & 1 & 0 \\
0 & 0 & 1 \\
1 & 0 & 0
\end{array}\right]^{3}\left[\begin{array}{ccc}
Q N_{1} & Q N_{0} & Q N_{-1} \\
Q N_{-1} & Q N_{-2} & Q N_{-3} \\
Q N_{0} & Q N_{-1} & Q N_{-2}
\end{array}\right]=\left[\begin{array}{ccc}
2 & 1 & 1 \\
1 & 1 & 0 \\
1 & 1 & 1
\end{array}\right]\left[\begin{array}{ccc}
Q N_{1} & Q N_{0} & Q N_{-1} \\
Q N_{-1} & Q N_{-2} & Q N_{-3} \\
Q N_{0} & Q N_{-1} & Q N_{-2}
\end{array}\right]} \\
=\left[\begin{array}{ccc}
2 Q N_{1}+Q N_{-1}+Q N_{0} & 2 Q N_{0}+Q N_{-2}+Q N_{-1} & 2 Q N_{-1}+Q N_{-3}+Q N_{-2} \\
Q N_{1}+Q N_{-1} & Q N_{0}+Q N_{-2} & Q N_{-1}+Q N_{-3} \\
Q N_{1}+Q N_{-1}+Q N_{0} & Q N_{0}+Q N_{-2}+Q N_{-1} & Q N_{-1}+Q N_{-3}+Q N_{-2}
\end{array}\right] \\
=\left[\begin{array}{lll}
Q N_{4} & Q N_{3} & Q N_{2} \\
Q N_{2} & Q N_{1} & Q N_{0} \\
Q N_{3} & Q N_{2} & Q N_{1}
\end{array}\right] .
\end{array}
$$

Assim, assumindo que vale para qualquer $n=k, k \in \mathbb{N}$ :

$$
\left[\begin{array}{lll}
1 & 1 & 0 \\
0 & 0 & 1 \\
1 & 0 & 0
\end{array}\right]^{k}\left[\begin{array}{ccc}
Q N_{1} & Q N_{0} & Q N_{-1} \\
Q N_{-1} & Q N_{-2} & Q N_{-3} \\
Q N_{0} & Q N_{-1} & Q N_{-2}
\end{array}\right]=\left[\begin{array}{ccc}
Q N_{k+1} & Q N_{k} & Q N_{k-1} \\
Q N_{k-1} & Q N_{k-2} & Q N_{k-3} \\
Q N_{k} & Q N_{k-1} & Q N_{k-2}
\end{array}\right] .
$$

Por fim, verifica-se a validade para $n=k+1$ :

$$
\left[\begin{array}{lll}
1 & 1 & 0 \\
0 & 0 & 1 \\
1 & 0 & 0
\end{array}\right]^{k+1}\left[\begin{array}{ccc}
Q N_{1} & Q N_{0} & Q N_{-1} \\
Q N_{-1} & Q N_{-2} & Q N_{-3} \\
Q N_{0} & Q N_{-1} & Q N_{-2}
\end{array}\right]
$$




$$
\begin{gathered}
=\left[\begin{array}{lll}
1 & 1 & 0 \\
0 & 0 & 1 \\
1 & 0 & 0
\end{array}\right]^{k}\left[\begin{array}{lll}
1 & 1 & 0 \\
0 & 0 & 1 \\
1 & 0 & 0
\end{array}\right]\left[\begin{array}{lll}
Q N_{1} & Q N_{0} & Q N_{-1} \\
Q N_{-1} & Q N_{-2} & Q N_{-3} \\
Q N_{0} & Q N_{-1} & Q N_{-2}
\end{array}\right] \\
=\left[\begin{array}{ccc}
N_{k+1} & N_{k} & N_{k-1} \\
N_{k-1} & N_{k-2} & N_{k-3} \\
N_{k} & N_{k-1} & N_{k-2}
\end{array}\right]\left[\begin{array}{lll}
1 & 1 & 0 \\
0 & 0 & 1 \\
1 & 0 & 0
\end{array}\right]\left[\begin{array}{lll}
Q N_{1} & Q N_{0} & Q N_{-1} \\
Q N_{-1} & Q N_{-2} & Q N_{-3} \\
Q N_{0} & Q N_{-1} & Q N_{-2}
\end{array}\right] \\
=\left[\begin{array}{cccc}
N_{k+2} & N_{k+1} & N_{k} \\
N_{k} & N_{k-1} & N_{k-2} \\
N_{k+1} & N_{k} & N_{k-1}
\end{array}\right]\left[\begin{array}{lll}
Q N_{1} & Q N_{0} & Q N_{-1} \\
Q N_{-1} & Q N_{-2} & Q N_{-3} \\
Q N_{0} & Q N_{-1} & Q N_{-2}
\end{array}\right] \\
=\left[\begin{array}{ccc}
Q N_{k+2} & Q N_{k+1} & Q N_{k} \\
Q N_{k} & Q N_{k-1} & Q N_{k-2} \\
Q N_{k+1} & Q N_{k} & Q N_{k-1}
\end{array}\right] .
\end{gathered}
$$

\section{A generalização dos quatérnios de Narayana}

Nessa seção, é realizada a generalização dos quatérnios de Narayana, ocorrendo uma extensão para os números inteiros não positivos.

Definição 3.1. Para todo $n>0$ e $n \in \mathbb{N}$, a fórmula de recorrência dos quatérnios de Narayana para índice inteiro não positivo, é dada por:

$$
Q N_{-n}=Q N_{-n+3}-Q N_{-n+2} \text {. }
$$

Definição 3.2. Para todo $n>0$ e $n \in \mathbb{N}$, o quatérnios de Narayana, para índice inteiro não positivo, é definido pela equação:

$$
Q N_{-n}=N_{-n}+i N_{-n+1}+j N_{-n+2}+k N_{-n+3}
$$

Propriedade 3.3. A função geradora dos quatérnios de Narayana para índice inteiro não positivo, é expressa por:

$$
g\left(Q N_{-n}, x\right)=\frac{i+j+k+(j+k) x-(1-i-j) x^{2}}{x^{3}-x^{2}-1},
$$

com os respectivos valores iniciais: $Q N_{-2}=1+k, Q N_{-1}=j+k$ e $Q N_{0}=i+j+k$.

ReviSeM, Ano 2021, No. 3, 12-22 
Demonstração. Realizando a multiplicação da função por $x^{2}$ e $x^{3}$, tem-se que:

$$
\begin{aligned}
g\left(Q N_{-n, x}\right) & =\sum_{n=0}^{\infty} Q N_{-n} x^{n}=Q N_{0}+Q N_{-1} x+Q N_{-2} x^{2}+\ldots+Q N_{-n} x^{n}+\ldots \\
x^{2} g\left(Q N_{-n}, x\right) & =Q N_{0} x^{2}+Q N_{-1} x^{3}+Q N_{-2} x^{4}+\ldots+Q N_{-n-2} x^{n}+\ldots \\
x^{3} g\left(Q N_{-n}, x\right) & =Q N_{0} x^{3}+Q N_{-1} x^{4}+Q N_{-2} x^{5}+\ldots+Q N_{-n-3} x^{n}+\ldots
\end{aligned}
$$

Assim, ao realizar a operação $x^{3} g\left(Q N_{-n}, x\right)-\left(x^{2} g\left(Q N_{-n}, x\right)+g\left(Q N_{-n}, x\right)\right)$, tem-se que:

$$
\begin{aligned}
\left(x^{3}-x^{2}-1\right) g\left(Q N_{-n}, x\right) & =Q N_{0}+Q N_{-1} x-\left(Q N_{-2}-Q N_{0}\right) x^{2} \\
\left(x^{3}-x^{2}-1\right) g\left(Q N_{-n}, x\right) & =i+j+k+(j+k) x-(1-i-j) x^{2} \\
g\left(Q N_{-n}, x\right) & =\frac{i+j+k+(j+k) x-(1-i-j) x^{2}}{x^{3}-x^{2}-1}
\end{aligned}
$$

Propriedade 3.4. Para $n>0$ e $n \in \mathbb{N}$, a matriz geradora dos quatérnios de Narayana, com indice inteiro não positivo, é dada por:

$$
\begin{aligned}
{\left[\begin{array}{ccc}
0 & 0 & 1 \\
1 & 0 & -1 \\
0 & 1 & 0
\end{array}\right]^{n}\left[\begin{array}{ccc}
Q N_{1} & Q N_{0} & Q N_{-1} \\
Q N_{-1} & Q N_{-2} & Q N_{-3} \\
Q N_{0} & Q N_{-1} & Q N_{-2}
\end{array}\right] } & =\left[\begin{array}{ccc}
N_{-n+1} & N_{-n} & N_{-n-1} \\
N_{-n-1} & N_{-n-2} & N_{-n-3} \\
N_{-n} & N_{-n-1} & N_{-n-2}
\end{array}\right]\left[\begin{array}{ccc}
Q N_{1} & Q N_{0} & Q N_{-1} \\
Q N_{-1} & Q N_{-2} & Q N_{-3} \\
Q N_{0} & Q N_{-1} & Q N_{-2}
\end{array}\right] \\
& =\left[\begin{array}{ccc}
Q N_{-n+1} & Q N_{-n} & Q N_{-n-1} \\
Q N_{-n-1} & Q N_{-n-2} & Q N_{-n-3} \\
Q N_{-n} & Q N_{-n-1} & Q N_{-n-2}
\end{array}\right] .
\end{aligned}
$$

Demonstração. De modo similar à demonstração realizada na Propriedade 2.5, pode-se validar a presente propriedade.

\section{Propriedades dos quatérnios de Narayana}

A seguir, são estudadas algumas propriedades inerentes aos quatérnios de Narayana.

Teorema 4.1. A soma dos n primeiros termos quatérnios de Narayana é dado por:

$$
\sum_{m=1}^{n} Q N_{m}=Q N_{n+3}-Q N_{3} .
$$


Demonstração. Utilizando a Definição 2.2, pode-se obter a relação:

$$
Q N_{n-3}=Q N_{n}-Q N_{n-1} \text {. }
$$

Assim,

$$
\begin{aligned}
Q N_{1} & =Q N_{4}-Q N_{3} \\
Q N_{2} & =Q N_{5}-Q N_{4} \\
Q N_{3} & =Q N_{6}-Q N_{5} \\
Q N_{4} & =Q N_{7}-Q N_{6} \\
Q N_{5} & =Q N_{8}-Q N_{7} \\
\vdots & \\
Q N_{n-3} & =Q N_{n}-Q N_{n-1} \\
Q N_{n-2} & =Q N_{n+1}-Q N_{n} \\
Q N_{n-1} & =Q N_{n+2}-Q N_{n+1} \\
Q N_{n} & =Q N_{n+3}-Q N_{n+2} .
\end{aligned}
$$

Após a realização dos cancelamentos sucessivos, tem-se que:

$$
\sum_{m=1}^{n} Q N_{m}=Q N_{n+3}-Q N_{3}
$$

Teorema 4.2. A soma dos 5 primeiros termos dos quatérnios de Narayana é dada por:

$$
\sum_{m=0}^{4} Q N_{m}=2 Q N_{n}+Q N_{n-1}
$$

Demonstração. Utilizando a Definição 2.2, tem-se:

$$
Q N_{n}+Q N_{n-1}+Q N_{n-2}+Q N_{n-3}+Q N_{n-4}=2 Q N_{n}+Q N_{n-1}
$$

Teorema 4.3. A soma dos $n$ primeiros termos, com índice inteiro não positivo, dos quatérnios de Narayana, são expressos por:

$$
\sum_{m=1}^{n} Q N_{-m}=Q N_{-n+3}+Q N_{2}
$$


Demonstração. Com base na relação de recorrência dos quatérnios de Narayana, com $n \in \mathbb{N}$, tem-se que:

$$
Q N_{-n}=Q N_{-n+3}-Q N_{-n+2} \text {. }
$$

Assim,

$$
\begin{aligned}
Q N_{-1} & =Q N_{2}-Q N_{1} \\
Q N_{-2} & =Q N_{1}-Q N_{0} \\
Q N_{-3} & =Q N_{0}-Q N_{-1} \\
Q N_{-4} & =Q N_{-1}-Q N_{-2} \\
\vdots & \\
Q N_{-n-3} & =Q N_{-n}-Q N_{-n-1} \\
Q N_{-n-2} & =Q N_{-n+1}-Q N_{-n} \\
Q N_{-n-1} & =Q N_{-n+2}-Q N_{-n+1} \\
Q N_{-n} & =Q N_{-n+3}-Q N_{-n+2} .
\end{aligned}
$$

Através de cancelamentos sucessivos, obtêm-se:

$$
\sum_{m=1}^{n} Q N_{-m}=Q N_{-n+3}+Q N_{2}
$$

Teorema 4.4. A soma dos 5 primeiros termos dos quatérnios de Narayana é dada por:

$$
\sum_{m=0}^{4} Q N_{m}=Q N_{-n+3}+2 Q N_{-n+4} .
$$

Demonstração. Com base na Definição 2.2, tem-se que:

$$
Q N_{-n}+Q N_{-n+1}+Q N_{-n+2}+Q N_{-n+3}+Q N_{-n+4}=Q N_{-n+3}+2 Q N_{-n+4} .
$$

\section{Conclusão}

Na presente pesquisa, foram introduzidos os quatérnios de Narayana, realizando um estudo matemático investigativo em torno da sua função geradora, fórmula de Binet, 
forma matricial e dentre outros aspectos matemáticos inerentes à essa sequência. Ao tudo, foi possível ainda realizar uma generalização para os números inteiros não positivos, obtendo outras propriedades.

Com a finalidade de ampliar os estudos de sequências lineares e recorrentes perante as suas aplicações, busca-se para estudos futuros, alternativas de integração dos quatérnios de Narayana com outras áreas afins, permitindo ainda a sua visualização computacional.

\section{Referências}

[1] Allouche, J. P.; Johnson, J. Narayana's cows and delayed morphisms, Articles of 3rd Computer Music Conference JIM96, France, 1996.

[2] Alves, F. R. V.; Vieira, R. P. M.; Catarino, P. M. M. C. Visualizing the Newtons Fractal from the Recurring Linear Sequence with Google Colab: An Example of Brazil X Portugal Research, International Electronic Journal of Mathematics Education, vol. 15, n. 3, p. 1-19, 2020.

[3] Alves, F. R. V. The Quaterniontonic and Octoniontonic Fibonacci Cassini's Identity: An Historical Investigation with the Maple's Help. International Electronic Journal of Mathematics Education, v. 13, n. 3, p. 125-138, 2018.

[4] Diskaya, O.; Menken, H. On the split (s,t)-Padovan and (s,t)-Perrin quaternions. International Journal of Applied Mathematics and Informatics, v. 13, p. 25-28, 2019 .

[5] Flaut, C.; Shpakivskyi, V. On Generalized Fibonacci Quaternions and FibonacciNarayana Quaternions, Arxiv, p. 1-18, 2012.

[6] Halici, S. On Fibonacci Quaternions. Adv. Appl. Clifford Algebras, 22(2), 321-327, 2012.

[7] Halici, S. On Complex Fibonacci Quaternions. Adv. Appl. Clifford Algebras, 23, 105-112, 2013.

[8] Horadam, A. F. Quaternion Recurrence relations, Ulam Quarterly, vol. 2, p. 23-33, 1993.

[9] Menon, M. J. Sobre as origens das definições dos produtos escalar e vetorial. Revista Brasileira de Ensino de Física, v. 31, n. 2, 2305, p. 1-11, 2009. 
[10] Oliveira, R. R. de. Engenharia Didática sobre o Modelo de Complexificação da Sequência Generalizada de Fibonacci:Relações Recorrentes N-dimensionais e Representações Polinomiais e Matriciais. Dissertação de Mestrado Acadêmico em Ensino de Ciências e Matemática-Instituto Federal de Educação, Ciência e Tecnologia do Estado do Ceará (IFCE), 2018.

[11] Oliveira, R. R.; Alves, F. R. V. A Fórmula de Binet e representações matriciais para os Quaternions Complexos de Fibonacci. REVISTA THEMA, v. 15, n. 3, p. 860-875, 2018.

[12] Ramírez, J. L.; Sirvent, V. F. A note on the k-Narayana sequence, Annales Mathematicae et Informaticae, vol. 45, p. 91-105, 2015.

[13] Tasci, D. Padovan and Pell-Padovan quaternions. Journal of Science and Arts, v. 42, n. 1, p. 125-132, 2018.

Submetido em 15 de Dezembro de 2020. Aceito em 15 de Janeiro de 2021. 